\title{
Impact of COVID-19 on Patient Behavior to Stroke Symptoms in China
}

\author{
Lili Song $^{a, b}$ Menglu Ouyang ${ }^{b}$ Lingli Sun $^{a}$ Chen Chen ${ }^{c}$ Craig S. Anderson ${ }^{a} b, d$ \\ ${ }^{a}$ The George Institute for Global Health at Peking University Health Science Center, Beijing, China; ${ }^{\text {T} T h e ~ G e o r g e ~}$ \\ Institute for Global Health, Faculty of Medicine, University of New South Wales, Sydney, NSW, Australia; ' $S$ hanghai \\ East Hospital, Tongji University, Shanghai, China; ${ }^{d}$ Neurology Department, Royal Prince Alfred Hospital, Sydney \\ Health Partners, Sydney, NSW, Australia
}

\section{Keywords}

COVID-19 $\cdot$ Stroke $\cdot$ Patient behavior $\cdot$ Coronavirus

Dear Editor,

The COVID-19 outbreak has had a major impact across the whole of China, affecting all aspects of people's daily lives and triggered psychological problems of anxiety and panic [1]. During the peak period of the outbreak in February and March 2020, the implementation of national policies to avoid social gatherings and travel to high-risk areas [2] resulted in hospitals reducing outpatient clinics and many services were restricted to urgent cases. Consequently, clinical research abruptly ceased, hospitals suspended ethics committee submissions and their review, and few in-person participant assessments were conducted. China is now emerging from this difficult period, but the shadow of COVID-19 continues, as we have encountered during initiation of the INTEnsive ambulance-delivered blood pressure Reduction in hyperACute stroke Trial (INTERACT4) (ClinicalTrials.gov NCT03790800, and Chinese Trial Registry ChiCTR1900020534).

$\begin{aligned} & \text { karger@karger.com } \\ & \text { www.karger.com/ced }\end{aligned}$
Karger $\%$

As the effectiveness of reperfusion therapy in highly time-dependent, public health strategies encourage patients to urgently call an ambulance, so that they can present rapidly to hospital after the onset of symptoms suggestive of acute stroke, most popularly defined by the Face symmetry, Arm weakness, Speech slurring, and Time to call (FAST) tool. We wish to highlight how COVID-19 has jeopardized this approach by adversely influencing the behavior of patients with suspected stroke.

INTERACT4 is a multicenter, prospective, randomized, open, blinded outcome assessed, trial of prehospital, ambulance-administered, intensive blood pressure-lowering treatment in patients with FAST-positive presumed acute stroke within $2 \mathrm{~h}$ from last known well. We launched the study on March 8, 2020, and the first patient was enrolled 2 weeks later. Until May 7, 2020, 12 patients have met the eligibility criteria from 84 patients screened across 4 hospitals in expanding research networks in Shanghai and Chengdu. This screened eligibility rate (14\% [8/59]) in the Emergency Department of the project lead hospital in Shanghai is much lower than that recorded $(32 \%$ [8/25]) in the period before the pandemic (20 April to July 1, 2017). The main reason that patients were

Craig S. Anderson

The George Institute for Global Health, Level 10

King George V Building, PO Box M201, Missenden Road

Sydney, NSW 2050 (Australia)

canderson@georgeinstitute.org.au 
excluded according to the ambulance screening logs is "delayed time from symptom onset" (>2 h $[n=39])$. The percentage of exceeding the time window among excluded patients during the pandemic (59\% [30/51]) is higher than that recorded in a similar period before the pandemic $(41 \%$ [7/17]) at the lead hospital. These patients (or their family members) indicated that they postponed calling the emergency hotline due to concerns of "getting infected" from being exposed at a "high-risk" hospital, and their first reaction was to wait and hope that the symptom(s) would mitigate or disappear. Moreover, parallel screening logs at participating hospitals indicate that many patients with mild stroke-related deficits chose to travel to hospital in their own vehicle, again to reduce a perceived high risk of infection via the ambulance.

Although "time is brain" is critical for effective reperfusion therapy, COVID-19 has clearly impacted the behavior of patients in China, as inferred elsewhere [3], with the likelihood that this will have flow-on adverse effects in reducing the potential for recovery from stroke and increasing the impact of subsequent disability on families and society. Although various clinical guidelines for the management of stroke during the COVID-19 pandemic have been released [4], additional efforts are needed to reassure the population and encourage people to seek rapid access to stroke services in order to receive essential interventions.

\section{Conflict of Interest Statement}

C.S.A. holds a Senior Investigator Fellowship of the National Health and Medical Research Council of Australia and reports honoraria and travel reimbursement and grants from Takeda, China. The other authors have no conflicts of interest to declare.

\section{Funding Sources}

No funding sources to report for the manuscript.

\section{Author Contributions}

C.S.A. and L.S. contributed to the concept and rationale for the study. M.O. and L.S. wrote the first draft of the manuscript with inputs from C.S.A. All authors commented upon and approved the final version of the manuscript for publication.

\section{References}

Impact of COVID-19 on Stroke Patients in China
1 Qiu J, Shen B, Zhao M, Wang Z, Xie B, Xu Y. A nationwide survey of psychological distress among Chinese people in the COVID-19 epidemic: implications and policy recommendations. Gen Psychiatr. 2020;33.

2 ChinaCDC. Novel coronavirus pneumonia prevention guidance for public (in Chinese). 2020.
3 Kansagra AP, Goyal MS, Hamilton S, Albers GW. Collateral effect of COVID-19 on stroke evaluation in the United States. N Engl J Med. 2020.

4 NIS. Clinical guide for the management of stroke patients during the coronavirus pandemic. 2020. : https://www.england.nhs.uk/ coronavirus/wp-content/uploads/sites/ 52/2020/04/C0033-_Specialty-guide_-Strokeand-coronavirus-V1-update_16-April-003. pdf. 